Regular Paper

\title{
Development of a control system for support equipment in operation support system.
}

\author{
Yuma TAKEI*1 (Stu. Mem.) and Masaya WATADA*1 (Mem.)
}

\begin{abstract}
Lately, minimally invasive surgeries have been gaining popularity. Laparoscopic surgery is a type of minimally invasive surgeries and is known to minimize scars and reduce the damage of patients, which allows a quick recovery. However, since the surgeon performs surgery in the limited area in the abdominal cavity, the surgeon's advanced techniques are required for operating forceps. As a countermeasure, we are developing an operation support system. The operation support system assists the surgeon to manipulate the forceps in the limited area. This system includes a support equipment and a master-slave combined robotic forceps. In this research, we will develop a control system for the support equipment. The paper will particularly outline overview and development of the operation support system for medical operations using a forceps and evaluate results of three control systems. As a result of the evaluation, it was confirmed that the developed system satisfies three functions.
\end{abstract}

Keywords: surgical robot, control system, mechanics.

(Received: 21 July 2018, Revised: 30 December 2018)

\section{Introduction}

Lately, minimally invasive surgeries have been significantly developed [1]. Laparoscopic surgery is a type of minimally invasive surgeries. In this surgery, the surgeon makes several $10 \mathrm{~mm}$ holes in the patient's body. The surgeon uses surgical instruments such as a forceps and a laparoscope which are thin enough to pass the small hole. This surgery requires only a few small holes. Therefore, scars from the operation are not noticeable. Moreover, the patient is able to recover quickly. However, the limited working space of a forceps disturbs the surgeon's action in the surgery. Therefore, this surgery is a huge burden on the surgeon. As a countermeasure, a number of laboratories have developed surgical robots [2].

We are developing the operation support system [3]. The operation support system consists of a support equipment and a master-slave combined robotic forceps. The robotic forceps has motion mechanism at the forceps' endpoint. Furthermore, the master-slave combined robotic forceps has the force sense detection mechanism. The support equipment has three functions. Their functions are gravity-compensation function, maintain function, and power-assist function.

In this research, we will develop the control system for support equipment that satisfy three functions in order to complete the support equipment in the operation support system. This paper will describe outline of the operation support system and the support equipment. Furthermore, construction and evaluation results of the three function's control system will be described.

Correspondence: Yuma Takei, Major Biomedical Engineering, Tokyo City University, 1-28-1 tamazutsumi, Setagaya-ku, Tokyo 158-8557, Japan email: g1781417@tcu.ac.jp

${ }^{* 1}$ Tokyo City University

\section{Operation support system}

\subsection{Overview of operation support system}

The operation support system consists of a support equipment and a master-slave combined robotic forceps. Fig. 1 shows operation support system.

The master-slave combined robotic forceps is a structure in which master (operation unit) and slave (working unit) are integrated. The master-slave combined robotic forceps' endpoint is capable of being driven. Therefore, the freedom of movement in the abdominal cavity increases. Furthermore, this forceps can detect reaction force when gripping an object. Therefore, it is possible to prevent organs from being crushed by applying excessive

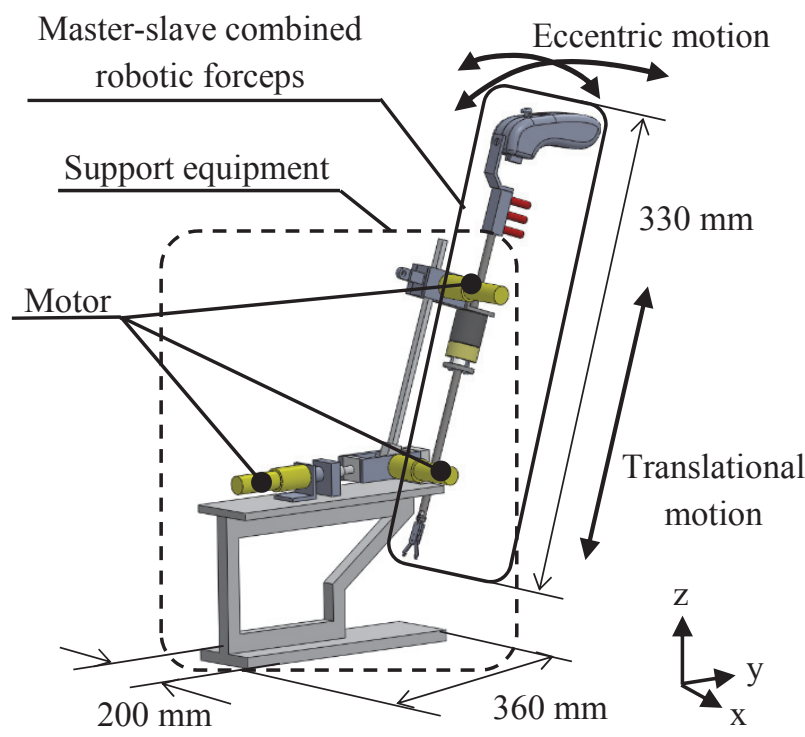

Fig. 1 Operation support system.

(Master-slave combined robotic forceps and Support equipment) 
force. With these functions, it is possible to solve the problems in the laparoscopic surgery [1]. As a result, the master-slave combined robotic forceps reduces surgeon's stress.

However, motors are mounted on the master-slave combined robotic forceps. Therefore, compared to laparoscopic surgical forceps, the weight of master-slave combined robotic forceps is heavy. As a result, the surgeon is affected by the weight of the forceps, and the surgeon stresses his arm. It is necessary to support surgery and reduce stress on the surgeon's arms. The support equipment will assist the operation. In this research, we will focus on the support equipment.

\subsection{Overview of Support equipment}

Fig. 2 shows support equipment. The support equipment consists of a robot arm and sensing device. The sensing device detects the force to operate the forceps. In this paper, we define this force as operating force. Depending on the detected operating force, the robot arm changes the motion. The sensing device uses 3-axis force sensor (TL3B05, by Tec Gihan Co, Ltd). Driving the robot arm, the support equipment assists operating force. The Digital Signal Processor (DSP) for controlling the support equipment uses s-BOX (by MTT, Ltd). Fig. 3 shows implementation platforms of the support equipment.

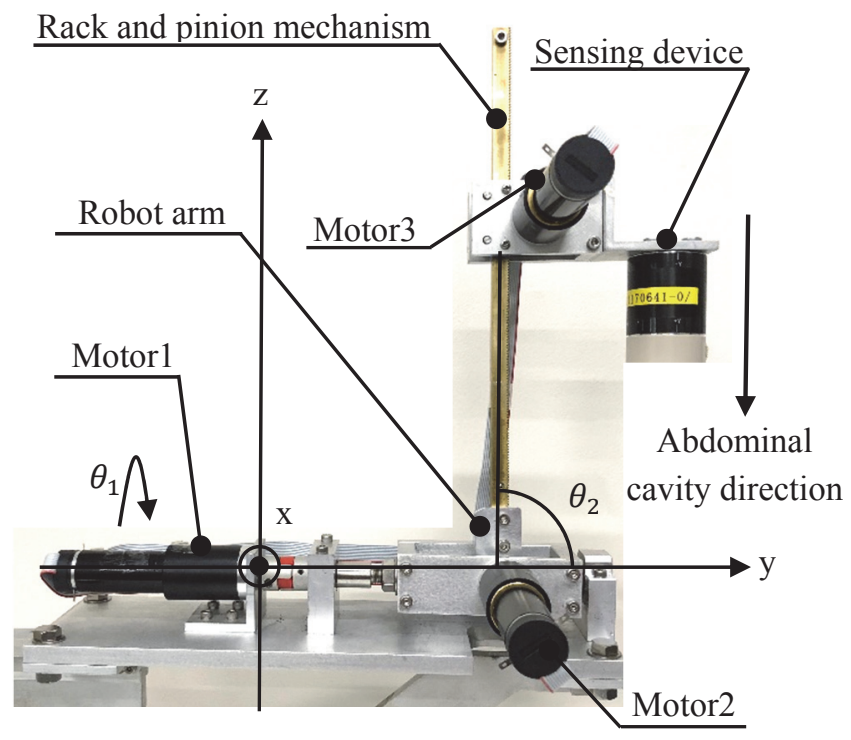

Fig. 2 Support equipment.

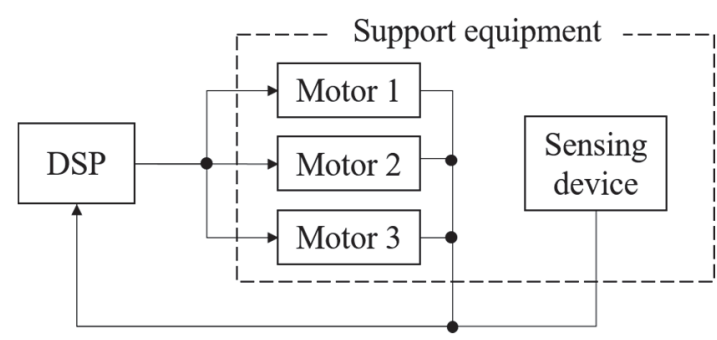

Fig. 3 Implementation platforms of support equipment.
Table 1 The required movable range and motor angle.

\begin{tabular}{|c|c|c|c|}
\hline \multicolumn{2}{|c|}{ Parameter } & Unit & Value \\
\hline \multirow{3}{*}{$\begin{array}{c}\text { Required } \\
\text { movable } \\
\text { range }\end{array}$} & Translational motion & $\mathrm{mm}$ & 200 \\
\cline { 2 - 4 } & $\begin{array}{c}\text { x-axis eccentric } \\
\text { motion }\end{array}$ & $\operatorname{deg}$ & \pm 45 \\
\cline { 2 - 4 } & $\begin{array}{c}\text { y-axis eccentric } \\
\text { motion }\end{array}$ & $\operatorname{deg}$ & \pm 45 \\
\hline \multirow{2}{*}{$\begin{array}{c}\text { Motor } \\
\text { angle }\end{array}$} & Motor1 angle: $\theta_{1}$ & $\operatorname{deg}$ & $-45 \sim 45$ \\
\cline { 2 - 4 } & Motor2 angle: $\theta_{2}$ & $\operatorname{deg}$ & $45 \sim 135$ \\
\cline { 2 - 4 } & Motor3 angle: $\theta_{3}$ & $\operatorname{deg}$ & $-360 \sim 360$ \\
\hline
\end{tabular}

For laparoscopic surgery, \pm 45 degrees eccentric motion to the normal direction of the abdominal wall and $200 \mathrm{~mm}$ translational motion are required for operation of forceps during the surgery [4]. Therefore, the support equipment performs the motion of which eccentricity is two degrees of freedom and of which translation is one degree of freedom. The support equipment's motion assists the surgeon's operation. Table 1 shows the required movable range and motor angle. The support equipment drives in the operation movable range shown in Table 1. Therefore, the support equipment realizes the required motion.

Driving the motor 1, the support equipment performs an eccentric motion in the $y$-axis direction. Driving the motor 2, the support equipment performs an eccentric motion in the $\mathrm{x}$-axis direction. The translational motion of the support equipment uses the rack and pinion mechanism. Driving the motor 3 , the support equipment performs translational motion. The direction of the translational motion is set to the abdominal cavity direction with reference to the abdominal wall. Furthermore, the $100 \mathrm{~mm}$ translational distance corresponds to $360 \mathrm{deg}$ angle of the motor 3 . The support equipment is driven by DC motor (RE25+GP32A+MR, by Maxon motor, Ltd). Motor controller uses driver (4-Q-DC Servo Control LSC30/2, by Maxon motor, Ltd).

\subsection{Control system for support equipment}

The support equipment has three functions. Their functions are gravity-compensation function, maintain function, and power-assist function [5].

Gravity-compensation function relieves the weight of the master-slave combined robotic forceps when the surgeon operates forceps. When relieving the weight of forceps, the surgeon is not affected by weight of forceps. The gravity-compensation function control system uses the value of the forceps' mass and each motor angle. Furthermore, the system calculates the load torque by solving the inverse dynamics equation (1).

$$
\begin{gathered}
\tau_{\text {load }}=\frac{d}{d t} \frac{\partial L}{\partial \dot{\theta}}-\frac{\partial L}{\partial \theta} \\
\tau_{\text {load }}: \text { Load torque }[\mathrm{Nm}] \quad L: \text { Lagrangian }[\mathrm{J}] \\
\theta: \text { Motor angle }[\mathrm{deg}]
\end{gathered}
$$




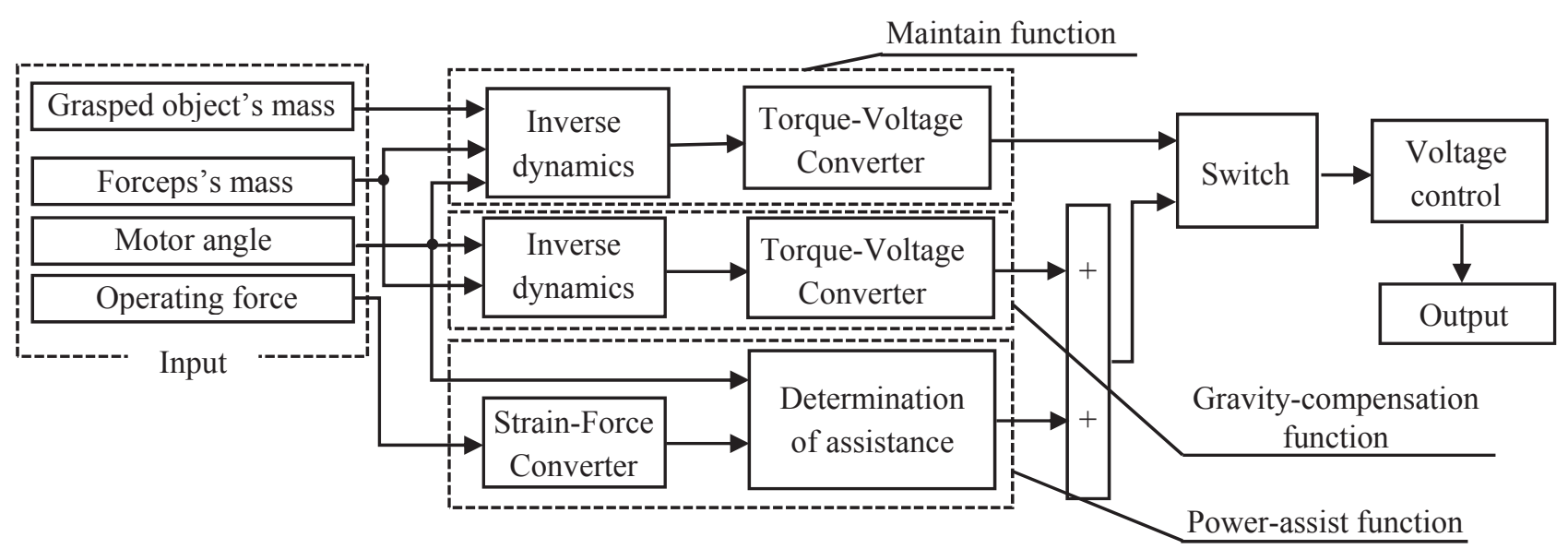

Fig. 4 Sequence diagram of the control system for support equipment.

It is possible to output the torque that cancels the load torque calculated by the inverse dynamics and to relieve the weight of the forceps.

The maintain function holds the position and posture of the master-slave combined robotic forceps when the surgeon releases his hand from the forceps. When holding the position and posture of the forceps, the surgeon can use the forceps as the third hand. The surgeon does not have to keep the forceps to hold the gripped object. The maintain function control system uses the value of the forceps mass and grasped object's mass and each motor angle. The load torque is calculated by solving the inverse dynamics equation of equation (1). It is possible to output the torque that cancels the load torque calculated by the inverse dynamics and to relieve the weight of the forceps and grasped object.

Power-assist function assists the surgeon's operating force when the surgeon operates the master-slave combined robotic forceps. When assisting surgeon's operating force, the surgeon operates the master-slave combined forceps with low operating force. The power-assist function control system uses the value of the operating force and each motor angle. The robot arm performs the eccentric motion and the translational motion following the operating force to the forceps [6].

In order for the support equipment to reduce the surgeon's stress, it is necessary to develop a control system. This system was constructed using integrated-development-environment (MATLAB/Simulink, by MathWorks, Ltd). Fig. 4 shows sequence diagram of the control system for support equipment.

\section{Evaluation of gravity-compensation function control system}

\subsection{Evaluation method of gravity-compensation func- tion control system}

We will evaluate the gravity-compensation function control system. The evaluation method is as below.

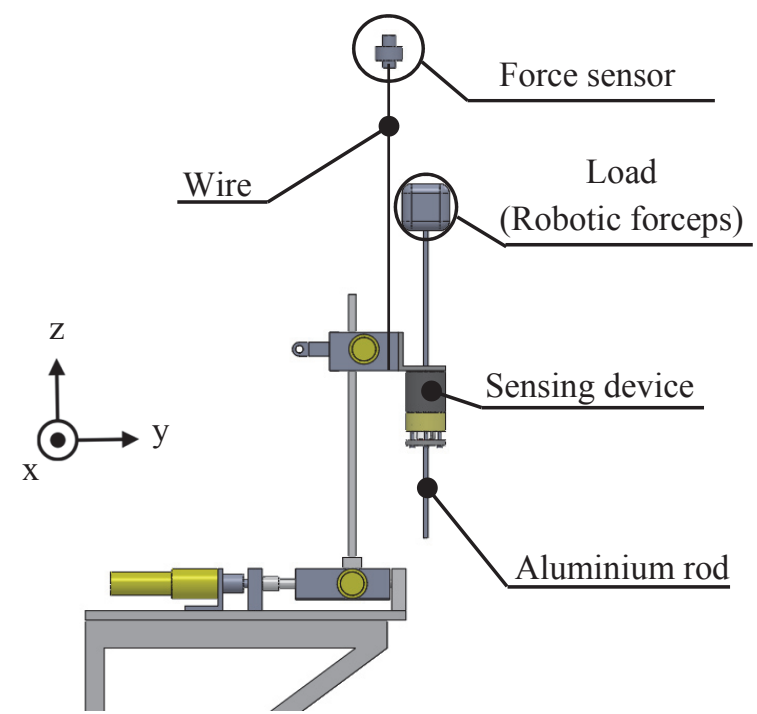

Fig. 5 Gravity-compensation function control system setup.

Table 2 Measurement condition.

(Gravity-compensation function control system)

\begin{tabular}{|c|c|c|c|}
\hline \multicolumn{2}{|c|}{ Parameter } & Unit & Value \\
\hline \multicolumn{2}{|c|}{ Range } & $\mu \varepsilon$ & 200 \\
\hline \multicolumn{2}{|c|}{ Sampling frequency } & $\mathrm{Hz}$ & 100 \\
\hline \multicolumn{2}{|c|}{ Load } & $\mathrm{g}$ & 300 \\
\hline \multirow{3}{*}{ Motor angle } & Motor1 angle: $\theta_{1}$ & deg & 0 \\
\hline & Motor 2 angle: $\theta_{2}$ & deg & 90 \\
\hline & Motor3 angle: $\theta_{3}$ & deg & 360 \\
\hline
\end{tabular}

Fig. 5 shows measurement device (gravity-compensation function control system) and Table 2 shows Mmeasurement condition (gravity-compensation function control system). We connected the wire to sensing device of the support equipment and hung it from the top. Furthermore, the top of the wire was connected to the 1axis force sensor (LUR-A-1KNSA1, by Kyowa electronic instruments, Ltd). The load object (300 g) was 
connected to the top of the aluminium rod connected to sensing device. The load object had the same mass as the master-slave combined robotic forceps. The translational distance was set to the position of the top dead point. The system was executed after changing command value to relieve $100 \sim 200 \%$ loading weight. Each value was increased by $50 \%$. The command value is derived by calculating the setting (2) by using the load torque of equation (1).

$$
\tau_{\text {out }}=\frac{\text { Rate }}{100} \times \tau_{\text {load }}
$$

$\tau_{\text {out }}$ : Output torque $[\mathrm{Nm}]$ Rate : Command value [\%]

$$
\tau_{\text {load }}: \text { Load torque }[\mathrm{Nm}]
$$

The command value was set on MATLAB/Simulink. The motor voltage was controlled so that the system realized the calculated output torque.

The gravity compensation function control system was carried out 5 seconds after the measurement started. We adjusted the zero point of the force sensor before connecting the load object.

We will evaluate loading weight detected by the force sensor when executing the gravity-compensation function control system. Force sensor is measured by measurement device (PCD301-B-F, by Kyowa electronic instruments, Ltd).

\subsection{Evaluation result of gravity-compensation function control system}

Fig. 6 shows the result of executing gravity-compensation function control system. According to Fig. 6, loading weight detected by the force sensor decreased when executing the gravity-compensation function control system. Therefore, it is possible to relieve the loading weight by the gravity-compensation function control system. However, the loading weight detected by the force sensor didn't reach $0 \mathrm{~N}$ when executing gravity compensation function control system with the command value of

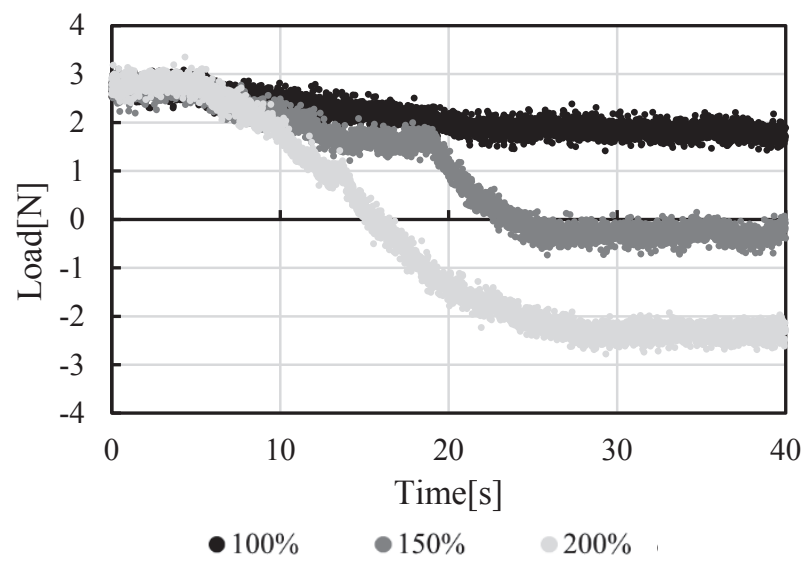

Fig. 6 The result of executing gravity-compensation function control system.
$100 \%$. Therefore, we confirm that the weight is not relieved in accordance with the command value. We consider that it is caused by mechanical loss between the pinion gear and the rack. The support equipment converts rotational motion into translational motion by the rack and pinion mechanism. We consider that the friction between the rack and pinion attenuated the motor torque [7]. Therefore, it is necessary to reduce the mechanical loss. In addition, the loading weight detected by the force sensor decreased with a rise in command value. In other words, when increasing the command value, it is possible to compensate for the attenuation of the motor torque caused by the mechanical loss. In addition, it is possible to change the rate of relief when command value is changed. Therefore, it is possible to set the rate at which the surgeon arbitrarily compensates.

\section{Evaluation of maintain function control system}

\subsection{Evaluation method of maintain function control sys- tem}

We will evaluate the maintain function control system. The evaluation method is as below.

Fig. 7 shows measurement device (maintain function control system) and Table 3 shows measurement condition (maintain control function control system). An

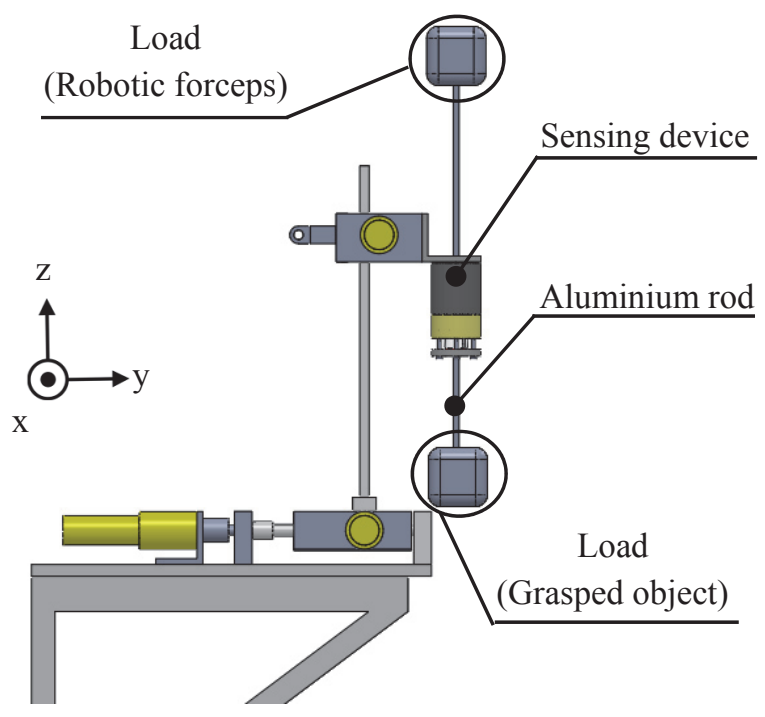

Fig. 7 Maintain function control system setup.

Table 3 Measurement condition.

(Maintain function control system)

\begin{tabular}{|c|c|c|c|}
\hline \multicolumn{2}{|c|}{ Parameter } & Unit & Value \\
\hline \multicolumn{2}{|c|}{ Sampling frequency } & $\mathrm{Hz}$ & 1000 \\
\hline \multirow{2}{*}{ Load } & Robotics forceps & $\mathrm{g}$ & 300 \\
\cline { 2 - 4 } & Grasped object & $\mathrm{g}$ & 300 \\
\hline \multirow{3}{*}{ Motor angle } & Motor1 angle: $\theta_{1}$ & $\mathrm{deg}$ & 0 \\
\cline { 2 - 4 } & Motor2 angle: $\theta_{2}$ & $\mathrm{deg}$ & 90 \\
\cline { 2 - 4 } & Motor3 angle: $\theta_{3}$ & $\mathrm{deg}$ & 360 \\
\hline
\end{tabular}




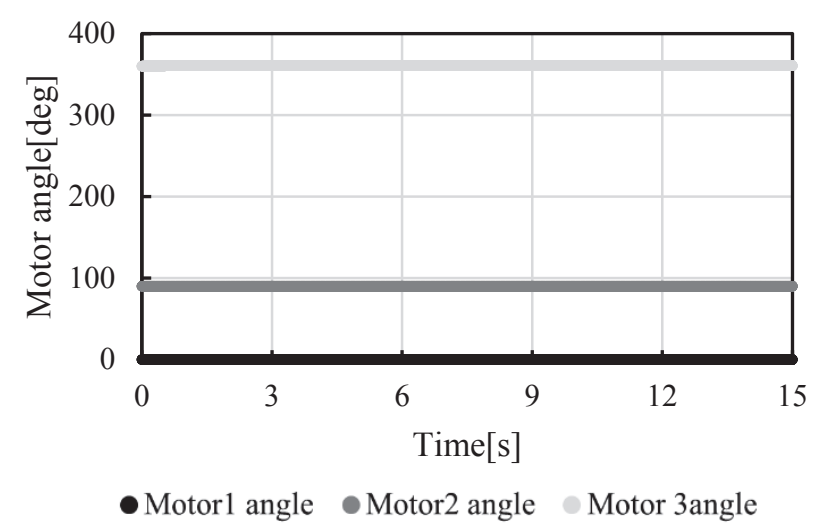

Fig. 8 The result of executing maintain function control system.

aluminium rod was connected to the sensing device of the support equipment, and a load object (300 g) was connected to its top. A load object had the same mass as the master-slave combined robotic forceps. Furthermore, we connected the lower end of the aluminium rod to the load object (300 g) assumed as the grasped object. The translational distance is set to the position of the top dead point. The evaluation device was the same as in section 3.2. We will evaluate each motor angle detected by the encoder in executing maintain function control system.

\subsection{Evaluation result of maintain function control sys- tem}

Fig. 8 shows the result of executing maintain function control system. According to Fig. 8, each motor angle was constant in executing the maintain function control system. Therefore, we confirmed that the support equipment holds its own posture. Furthermore, similar results were confirmed within the operating range of the support equipment by the same experimental method. Therefore, it is possible to hold the position and posture of the forceps by maintain function control system.

\section{Evaluation of power-assist function control system}

\subsection{Evaluation method of power-assist function control system}

We will evaluate power-assist function control system. The evaluation method is as below.

Fig. 9 shows measurement device (power-assist function control system) and Table 4 shows measurement condition (power-assist function control system). An aluminum rod was connected to the sensing device of the support equipment, and a load object (300 g) was connected to the upper end. A load object has the same mass as the master-slave combined robotic forceps. Furthermore, we attached the 1-axis force sensor to the load object. The translational distance is set to the position of the top dead point.

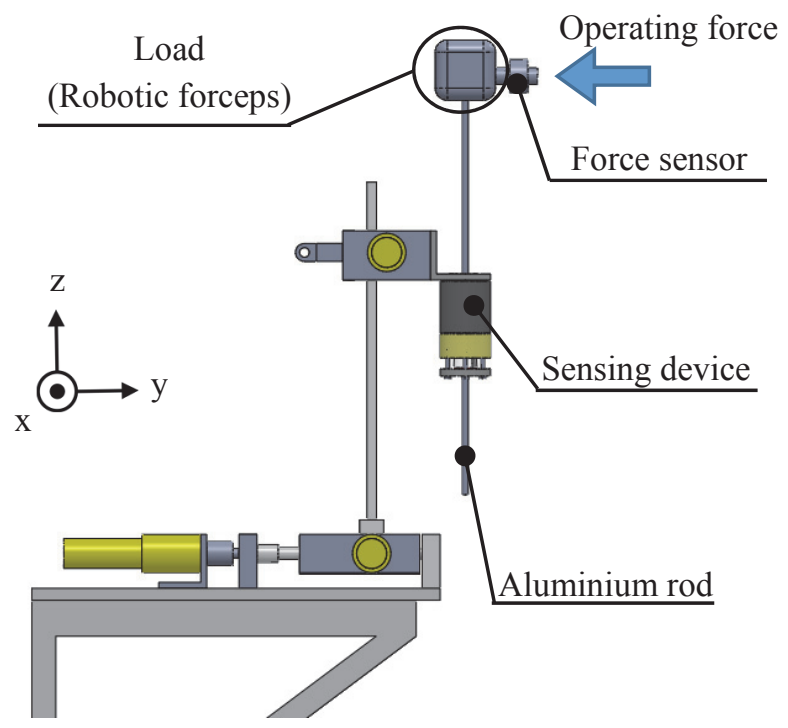

Fig. 9 Power-assist function control system setup.

Table 4 Measurement condition.

(Power-assist function control system)

\begin{tabular}{|c|c|c|c|}
\hline \multicolumn{2}{|c|}{ Parameter } & Unit & Value \\
\hline \multicolumn{2}{|c|}{ Range } & $\mu \varepsilon$ & 200 \\
\hline \multicolumn{2}{|c|}{ Sampling frequency } & $\mathrm{Hz}$ & 100 \\
\hline \multicolumn{2}{|c|}{ Load } & $\mathrm{g}$ & 300 \\
\hline \multirow{3}{*}{ Motor angle } & Motor1 angle: $\theta_{1}$ & deg & $-45 \sim 0$ \\
\hline & Motor2 angle: $\theta_{2}$ & deg & $45 \sim 90$ \\
\hline & Motor3 angle: $\theta_{3}$ & deg & 360 \\
\hline
\end{tabular}

In the above described state, the support equipment performed the $\mathrm{y}$-axis eccentric motion and the $\mathrm{x}$-axis eccentric motion. Each motion performed by pushing the 1axis force sensor was connected to the load object by hand. The power-assist function control system was carried out 5 seconds after the measurement started. The evaluation device was the same as in Section 3.2. 3-axis force sensor was measured by measurement device (PCD301-B-F, by Kyowa electronic instruments, Ltd). 1axis force sensor was measured by measurement device (PCD300-A, by Kyowa electronic instruments, Ltd). The value detected by sensing device was multiplied constantly by 0 to 0.6 . Each value was increased by 0.1 . The voltage amplification value was set on MATLAB/Simulink. We will evaluate operating force detected by the force sensor when executing power-assist function control system.

\subsection{Evaluation result of power-assist function control system}

Fig. 10 shows the result of executing the power-assist function control system ( $y$ - axis eccentric motion) and Table 5 shows Max Operating force value (y-axis eccentric 


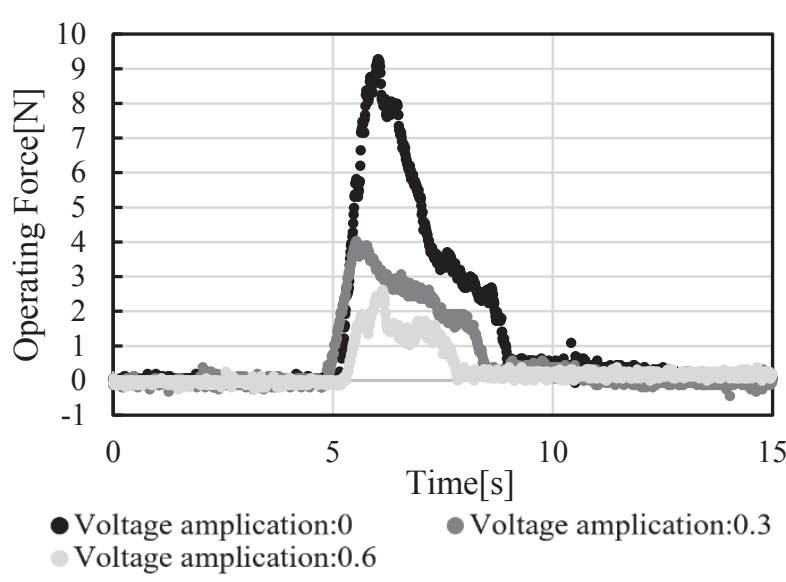

Fig. 10 The result of executing power-assist function control system. (y-axis eccentric motion)

Table 5 Max Operating force value. (y-axis eccentric motion)

\begin{tabular}{|c|c|c|}
\hline \multirow{2}{*}{ Parameter } & & Value(N) \\
\hline \multirow{4}{*}{$\begin{array}{c}\text { Voltage } \\
\text { Amplification value }\end{array}$} & 0 & 9.26 \\
\cline { 2 - 3 } & 0.1 & 6.71 \\
\cline { 2 - 3 } & 0.2 & 4.47 \\
\cline { 2 - 3 } & 0.3 & 4.02 \\
\cline { 2 - 3 } & 0.4 & 3.70 \\
\cline { 2 - 3 } & 0.5 & 3.00 \\
\cline { 2 - 3 } & 0.6 & 2.62 \\
\hline
\end{tabular}

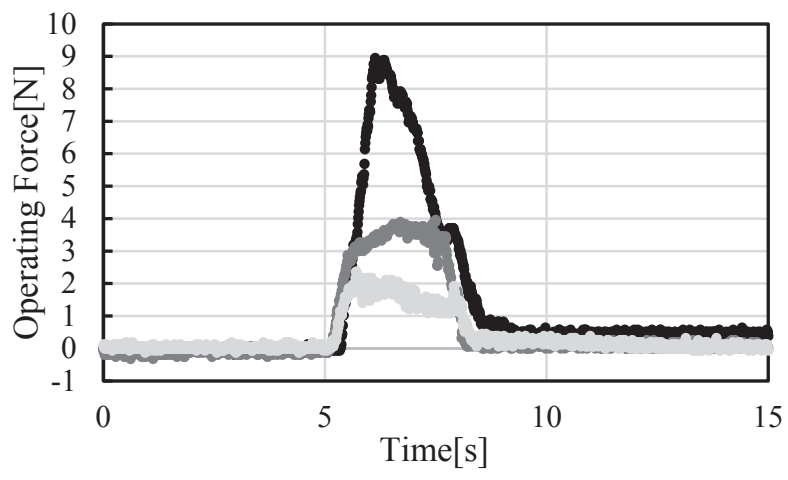

- Voltage amplication:0 Voltage amplication: 0.6

Fig. 11 The result of executing power-assist function control system. (x-axis eccentric motion)

Table 6 Max Operating force value.

(x-axis eccentric motion)

\begin{tabular}{|c|c|c|}
\hline \multicolumn{2}{|c|}{ Parameter } & Value(N) \\
\hline \multirow{7}{*}{$\begin{array}{c}\text { Voltage } \\
\text { Amplification value }\end{array}$} & 0 & 8.95 \\
\hline & 0.1 & 7.54 \\
\hline & 0.2 & 5.75 \\
\hline & 0.3 & 3.96 \\
\hline & 0.4 & 3.04 \\
\hline & 0.5 & 2.81 \\
\hline & 0.6 & 2.55 \\
\hline
\end{tabular}

motion). In addition, Fig. 11 shows the result of executing power-assist function control system ( $\mathrm{x}$-axis eccentric motion) and Table 6 shows Max Operating force value (x-axis eccentric motion).

According to Fig. 10 and Table 5, in the y-axis eccentric operation, the operating force detected by the force sensor decreased as the voltage amplification value increased. Furthermore, we also confirmed similar results in $\mathrm{X}$-axis eccentric motion. In other words, power-assist system assists operating force. Therefore, it is possible to assist surgeon's operating force by power-assist function control system.

\section{Conclusion}

In this paper, we described evaluation of the control system for the support equipment. The support equipment has three functions: gravity-compensation function, maintain function, and power-assist function.

As a result of executing gravity compensation function control system, we confirmed that it is possible to relieve the loading weight by this control system. In addition, we confirmed that it is possible to change the rate of relief by changing the command value. Furthermore, as a result of executing maintain function control system, we confirmed that it is possible to hold the position and posture of the forceps by this control system. As a result of executing power-assist function system, we confirmed that it is possible to assist the surgeon's operating force by this control system. Therefore, three functions mounted on the support equipment were realized.

In the future, we will evaluate the control system dynamically and qualitatively in the situation where the surgeon uses the support equipment.

\section{References}

[1] D. Hashimoto, "Current problems and its solutions in minimally invasive surgery," Modern media, Vol. 50, No. 10, pp. 233-240, 2004.

[2] D. Jinno, "Robotic forceps for laparoscopic surgery," Journal of the Robotic Society of Japan, Vol. 22, No. 4, pp. 17-20, 2004.

[3] K. Shimizu and M. Watada, "The proposal of the HumanMachine Cooperative System for Master-Slave Combined Robotic forceps," APSAEM Conference, Vol. 19, No. 3, pp. 9-12, 2010.

[4] Y. Kobayashi, M. Okada and Y. Nakamura, "Slave Robot System that does not Occupy Large Space in Operating Rooms," Journal of the Robotic Society of Japan, Vol. 19, pp. 757-758, 2001

[5] Y. Takei, J. Kuriki, M. Watada, Y, Shoji and N. Matsubayashi, "Construction of a power-assist function system for support equipment in operation support system," MAGDA conference, Vol. 26, pp. 359-364, 2017.

[6] S. Hirano, S. Wakoh and K. Sagawa, "Development of Power-Assisted Wagon," The Japan Society of Mechanical Engineers IBARAKI CONFERENCE, pp. 263-264, 2000.

[7] Y. Takei, M. Watada, Y, Shoji and N. Matsubayashi, "Evaluation of performance by improvement of support equipment in operation support system," MAGDA conference, Vol. 27, pp. 442-447, 2018. 